\title{
Fabrication and characterisation of photonic nanowires
}

\author{
Joseph E McCarthy ${ }^{\mathrm{a}}$, Áine M Whelan ${ }^{\mathrm{a}}$, Gemma-Louise Davies ${ }^{\mathrm{a}}$, Fiona Byrne ${ }^{\mathrm{b}}$, Jennifer Conroy ${ }^{\mathrm{c}}$, \\ Yuri Volkov', Yurii Gun'ko ${ }^{\mathrm{a}}$ \\ ${ }^{a}$ School of Chemistry, Trinity College Dublin \\ ${ }^{b}$ School of Physics, Trinity College Dublin \\ ${ }^{c}$ Institute of Molecular Medicine, St. James' Hospital, Dublin
}

\begin{abstract}
In recent years the application of nano-porous templates, such as anodic alumina and PTFE, in the production of cylindrical nanostructures has been vast. In our work we used porous alumina membranes to produce luminescent nanowires from polystyrene and silica. The silica wires were fabricated by infiltration of a TEOS derived sol-gel into $200 \mathrm{~nm}$ diameter porous alumina membranes with vacuum assistance followed by annealing at $400{ }^{\circ} \mathrm{C}$. Polystyrene luminescent, magnetic nanowires have been fabricated using a similar technique. The wires were studied by optical, confocal and transmission electron microscopy. Silica nanowires demonstrated a broad luminescence spectrum due to interstitial carbon defect emission. Polystyrene nanowires have demonstrated strong emission and interesting magnetic behaviour. Both polystyrene and silica maghemite loaded nanowires show alignment to an external magnetic field. We believe that these silica and polystyrene nanowires might find potential applications in photonics, bio-sensing and biological imaging.
\end{abstract}

Keywords: Nanowires, Sol-gel, Polystyrene, nano-porous templates

\section{Introduction}

In recent years the need for the relatively cheap production of uniform nanoscale structures has been in great demand in all aspects of research, be it academic to industrial $\mathrm{R} \& \mathrm{D}$ to commercial product manufacture. The ready availability of nanosized porous templates (such as anodized alumina, macroporous $\mathrm{Si}$ and polytetrafluoroethylene (PTFE) membranes) has enabled such relatively cheap and easy production ${ }^{[1,2]}$. The use of templates to create 1-D linear assemblies has been incorporated to produce nanoparticle (NP) chains. Before this the chains were produced via NP interactions such as magnetic/electronic dipole moments and the use of couplers etc. However, this method of formation was not always ideal and only certain materials possessed the correct properties of this self-assembly growth. Structures such as DNA and carbon nanotubes (CNTs) were then employed as templates with great success in forming NP chains from a wide range of materials ${ }^{[3]}$. To further simplify 1D nano structure formation, infiltration into ordered hollow structures was the next obvious step and using easily manipulated and doped fluids such as sol-gels, polymer solutions and melts, NP properties can be utilised in this dimension with cost effective ease.

At micron and large nano scales viscosity, surface tension and interfacial energies of the solutions dominate and they do not necessarily behave like normal liquids. Therefore the liquid must be of the right viscosity to infiltrate the pores of the membrane; therefore in the case of polymers solutions an appropriate ratio of polymer to solvent is required. It has been well observed that polymer melts readily wet the walls of porous templates forming nanotubes ${ }^{[2]}$. However to achieve complete filling of the pores vacuum assist infiltration is required to break the surface tension and pull the solution completely into the pores.

Recently, there has been intense effort devoted to the application of one dimensional nanomaterials (nanowires) in a variety of bio-medical technologies. Extensive research has been carried out on nanoparticles in the field of bio-sensing, bio-imaging and bio-sorting. The majority of this work has explored the unique properties of luminescent materials especially semiconductor nano-materials such as II-VI quantum dots (CdTe). Magnetic properties of nano-materials

Nanophotonic Materials V, edited by Zeno Gaburro, Stefano Cabrini, Dmitri Talapin Proc. of SPIE Vol. 7030, 703019, (2008) · 0277-786X/08/\$18 · doi: 10.1117/12.810742 
have also come to the fore in bio-medical applications in recent years for applications in improving contrast in magnetic resonance imaging (MRI) ${ }^{[4]}$ and magnetic sorting of biological entities. Magnetic nanoparticles such as dynabeads have been extensively researched however it has been shown that one-dimensional nanowires can have a superior magnetic moment ${ }^{[5,6]}$. Magnetic nanowires have been shown to viable tools for immunoassays ${ }^{[7]}$ and magnetic sorting ${ }^{[5,8]}$. More specifically the application of iron oxide nanoparticles in these applications has been widespread due to their superparamagnetic properties and ready surface functionalisation allowing bio compatibility in a wide range of systems $^{[9,10]}$.

The main aims of the work were to fabricate one dimensional nanostructures using vacuum assisted solution infiltration of porous alumina membranes using silica sol-gels and polystyrene solutions and investigate them using various techniques such as optical microscopy and fluorescence optical microscopy, transmission electron microscopy (TEM), scanning electron microscopy (SEM) and vibrating sample magnetometry (VSM).

\section{Experimental}

Unless stated otherwise all chemicals were purchased from Sigma-Aldrich. All reactions with water involved were carried out using Millipore water. Magnetisation measurements were carried out at RT using a LakeShore 7300 vibrating sample magnetometer (VSM). SEM studies have been performed using a Hitachi S-4300 on gold coated surfaces. TEM images were obtained on a Hitachi H 7000 electron microscope operating at $100 \mathrm{kV}$. Fluorescent images were acquired by fluorescence microscopy (Nikon Eclipse TE 300).(Nikon Eclipse TE 2000-U). Photoluminescence spectra were taken using a Renishaw 1000 micro-Raman system equipped with a Leica microscope.

\section{Luminescent silica nanowires}

Tetraethoxysilane (TEOS) was hydrolysed for 2 hours using a solution of $\mathrm{H}_{2} \mathrm{O}$ and ethanol in a 1:1:1 molar ratio using conc. $\mathrm{HCl}\left(1\right.$ drop) as a catalyst. The condensation reaction was then performed using a solution of 4:1 $\mathrm{H}_{2} \mathrm{O}$ : ethanol and 3 drops of conc. $\mathrm{HCl}$ as catalyst. The sol was then allowed to stir for $\sim 20 \mathrm{mins}$ and infiltrated into $200 \mathrm{~nm}$ diameter porous alumina under vacuum assist using 2-3 drops of solution. The sample was allowed to dry in air for 3 days. Gelation time of the sol was about $48 \mathrm{~h}$. The infiltrated membrane was then placed in a furnace and heated to $400{ }^{\circ} \mathrm{C}$ at $5^{\circ} / \mathrm{min}$ and dwelled for $1 \mathrm{~h}$. The sample was then (after cooling) placed in $0.11 \mathrm{M} \mathrm{NaOH}$ solution with sonication to dissolve the membrane and release the wires. The suspended wires were then repeatedly washed via centrifugation with water until a neutral $\mathrm{pH}$ was achieved.

\section{Maghemite loaded silica nanowires}

TEOS was hydrolysed using a solution of $\mathrm{H}_{2} \mathrm{O}$, ethanol in a 1:1:1 ratio and using conc. $\mathrm{HCl}$ solution (1 drop) as a catalyst. The condensation reaction was carried out using a solution of $\mathrm{H}_{2} \mathrm{O}$, ethanol (4:1) and conc. $\mathrm{NH}_{3}$ solution (3 drops). At this time a solution of iron oxide nanoparticles coated with cetyl-trimethylammonium-bromide (CTAB) was added forming a homogenous sol. This was allowed stir for a few minutes and then infiltrated under vacuum assist into $200 \mathrm{~nm}$ diameter porous alumina membranes using 2-3 drops of solution. The gelation time of the sol was $\sim 6-7$ minutes. The samples were allowed to dry in air for 1 day. The wires were released from the membranes by polishing the surface using fine grade sand paper and placing in $0.11 \mathrm{M} \mathrm{NaOH}$. The wires were then washed with water via centrifugation as above. The wires were labeled with antibodies using Alexa 488 mouse IgM antibodies. This was done by firstly priming the surface with aminopropyltriethoxysilane (APTES) in isopropanol and left to mix on a vortex overnight. The wires were then redispersed in water and the linker EDC (1-ethyl-3-(3-dimethylaminopropyl) carbodiimide hydrochloride) was added along with the antibody.

\section{Fluorescent magnetic polystyrene nanowires}

$300 \mathrm{mg}$ of high molecular weight polystyrene $\left(\mathrm{M}_{\mathrm{w}}=350,000, \mathrm{M}_{\mathrm{n}}=170,000\right)$ was added to $10 \mathrm{ml}$ of tetrahydrofuran (THF) and treated with a sonic tip (power $=75 \mathrm{~W}$ ) for 10 mins to dissolve the polystyrene. To make the wires magnetic and fluorescent $0.6 \mathrm{ml}$ of Oleic acid coated magnetite in chloroform and $3 \mathrm{mg}$ of coumarin 153 were added. This created a fluorescent green tinted brown homogenous solution of suitable viscosity to be infiltrated into a $200 \mathrm{~nm}$ diameter porous alumina membrane with vacuum assistance. The membranes are then left to dry for $24 \mathrm{hrs}$ in air and then sealed in an air tight container. The surfaces of the membrane are then polished using fine grade sand paper and placed in $1 \mathrm{M}$ 
$\mathrm{NaOH}$ to dissolve the membrane and release the wires. This mixture was then centrifuged at $5000 \mathrm{rpm}$ in an eppendorf to bring the wires out of suspension and the $\mathrm{NaOH}$ was pippeted off and the wires washed with water, this process is repeated until a near neutral $\mathrm{pH}$ was achieved.

\section{Results and Discussion}

\section{Luminescent silica nanowires}

It has been previously reported by Green et al ${ }^{[11]}$ that TEOS derived silica gels exhibit visible luminescence under UV excitation via defects in the silica network. It has previously been reported the use of this phenomenon in the fabrication of silica microtubes ${ }^{[12,13]}$ with some success in achieving intense broad visible emission from the tubes. The thermal treatment of the nanowires in this case is thought to have given rise to substituted $\mathrm{Si}$ atoms with carbon due to the degradation of organic species causing a luminescent centre in the silica network. The origin of the carbon species is assumed to be unhydrolysed alkoxy groups trapped in the silica network during sol-gel processing. Figure 1 shows TEM images of the released wires and a fluorescence microscope image of the nanowires emitting. The wires exhibit high aspect ratio with dimensions of diameter $\sim 160 \mathrm{~nm}$ and lengths of several microns. Some of the wires also show deformations due to the alumina matrix as can be seen form Figure 1 (b) below. This is due to the sol-gel filling merged or deformed pores.

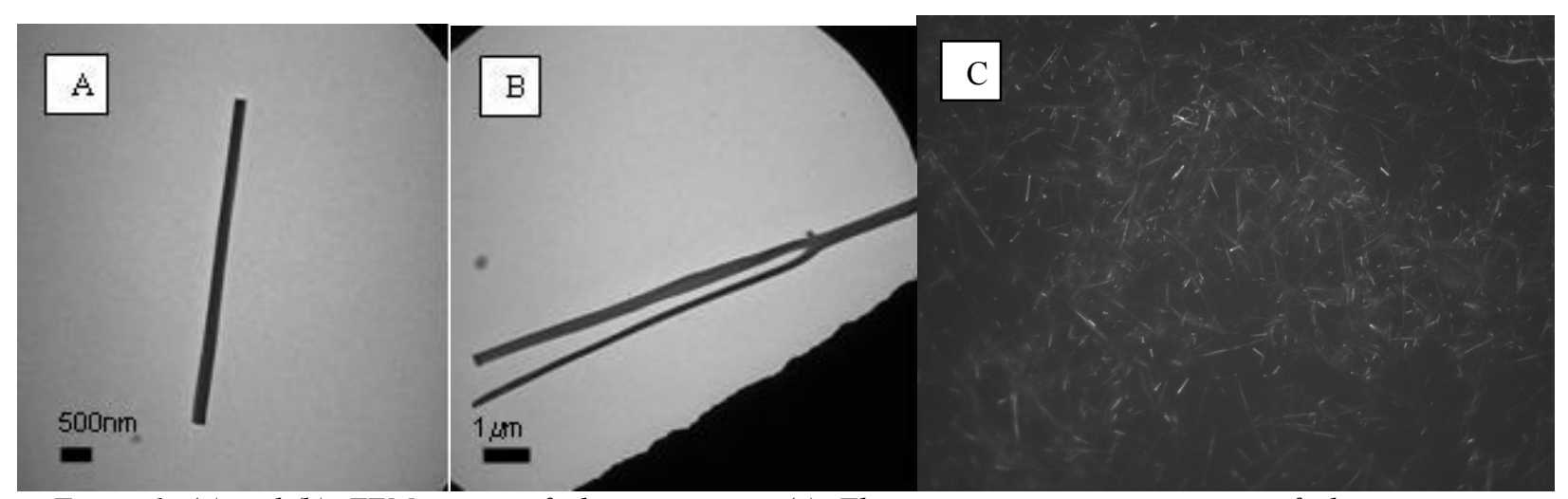

Figure 1: (a) and (b); TEM images of silica nanowires (c); Fluorescence microscope image of silica nanowires

A room temperature photoluminescence (PL) spectrum of bulk sol-gel made under the same experimental conditions and exposed to the same treatments is shown in Figure 2 below. The broad intense emission shown in the visible region is believed to be due to defect emission as discussed above.
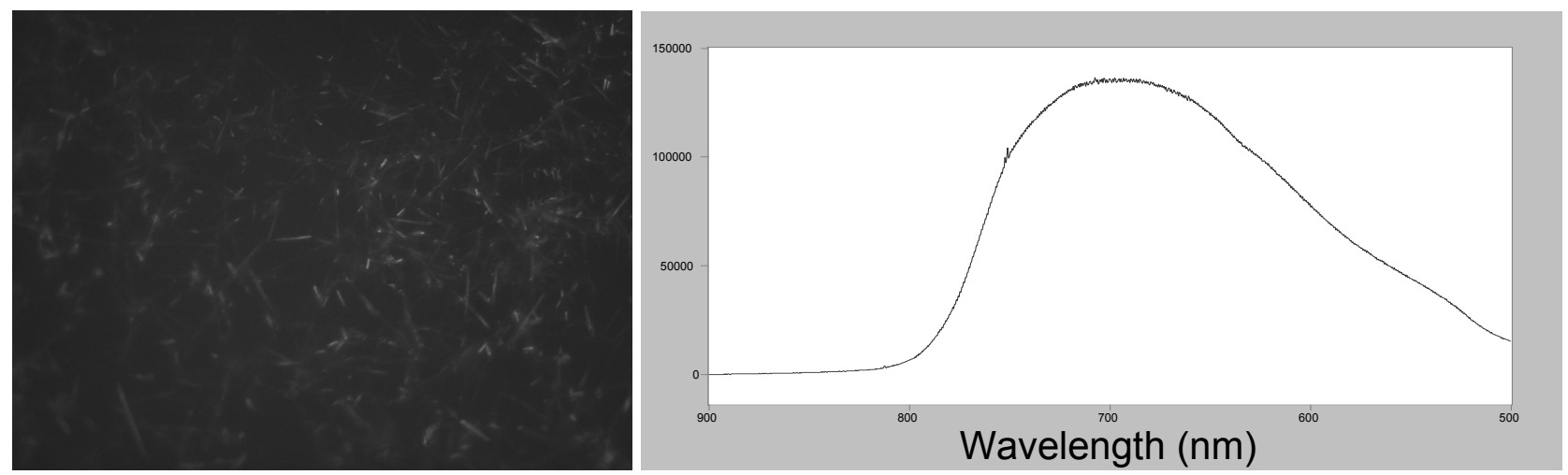

Figure 2: left; fluorescence microscope image of emitting nanowires, right; PL spectrum of bulk gel (ex457nm) 


\section{Maghemite loaded silica nanowires}

The fabrication of silica nanotubes containing magnetite nanoparticles within its hollow core has recently been reported ${ }^{[14]}$ however to the authors knowledge this is the first report of silica nanowires containing maghemite nanoparticles in the silica matrix. The superparamagnetic properties of iron oxides made them an ideal substance for the magnetic properties required in biological applications. As shown in Figure 3 (c), the nanowires do show this superparamagnetic property however it's much weaker than in the particles themselves with magnetisation saturation at $0.04 \mathrm{Am}^{2} / \mathrm{Kg}$. This is probably due to the conditions and use of acidic and basic catalysts in the sol-gel preparation. The wires are well formed within the pores with high aspect ratio and shrink off the pore walls with drying and shrinkage of the gel over time as can be seen in Figure 3 (a) below. The wires can also be preferentially aligned along a magnetic field showing their macro magnetic properties. Figure 3 (b) shows the loaded silica wires labelled with Alexa 488 mouse IgM antibodies showing that the wires are viable structures for tagging and immunoassays. Biological testing of these wires is currently being carried out.
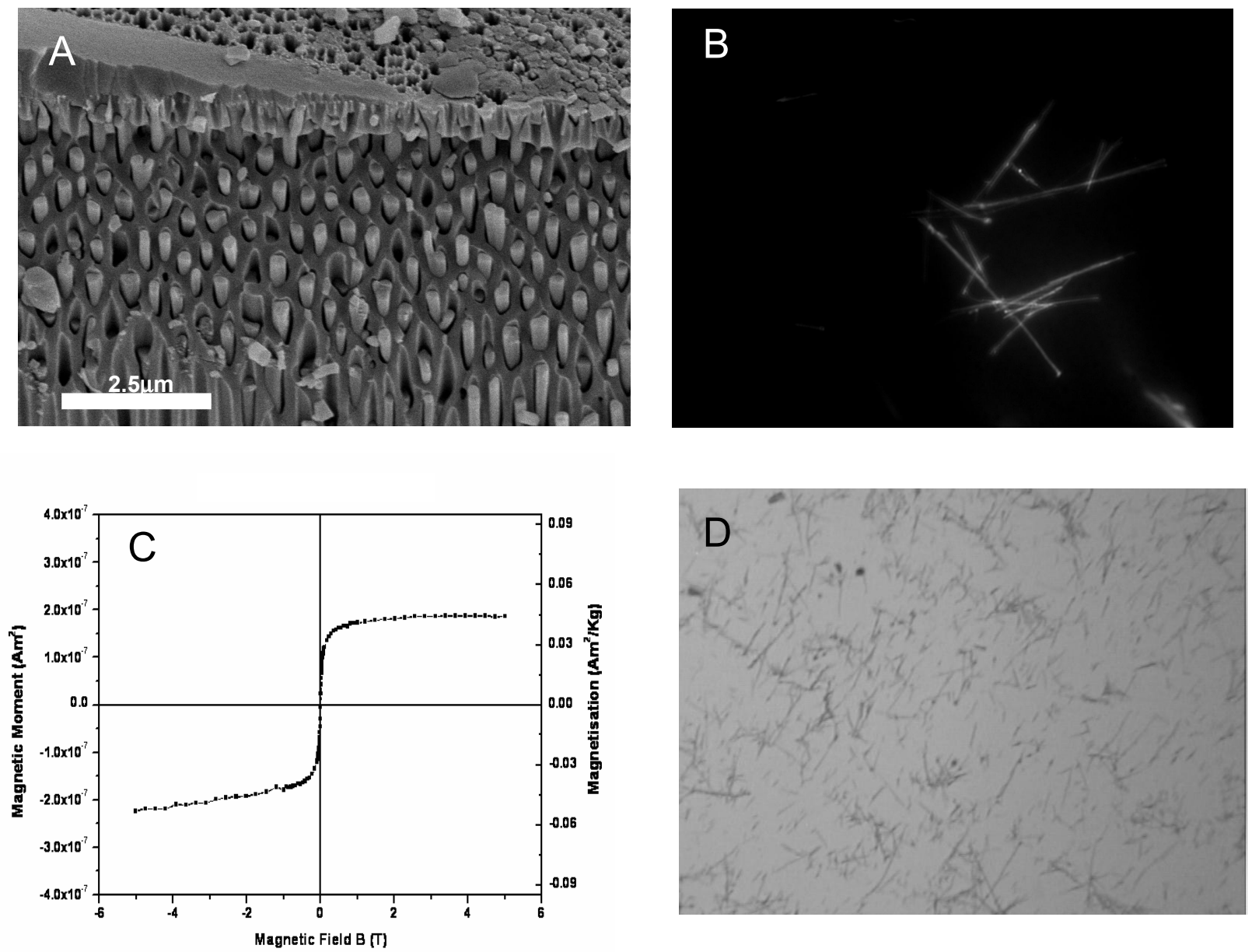

Figure 3: A: SEM image of fractured alumina membrane showing wires contained in pores; B: Confocal image of fluorescent silica nanowires; $\boldsymbol{C}$ : Magnetisation curve (VSM measurement) of maghemite loaded silica nanowires; D: optical microscope image of wires aligned in an external magnetic field.

\section{Fluorescent magnetic polystyrene nanowires}

Figure 4 below shows SEM images of standing arrays of polystyrene nanowires. The wires show high aspect ratio with a diameter of just under $200 \mathrm{~nm}$ (slightly under alumina pore size after some shrinkage during drying) and lengths of a few microns. The magnetic properties of the polystyrene doped with iron oxide nanoparticles are shown in Figure 4 
(right) below and show a magnetisation saturation of $\sim 3 \mathrm{Am}^{2} / \mathrm{Kg}$. This is demonstrated visually in the nanowires in the fluorescence microscope images shown below in Figure 5. The wires show very good alignment to the applied external magnetic field and can be moved and manipulated in solution using a magnetic field. The wires also show strong luminescence under UV excitation.
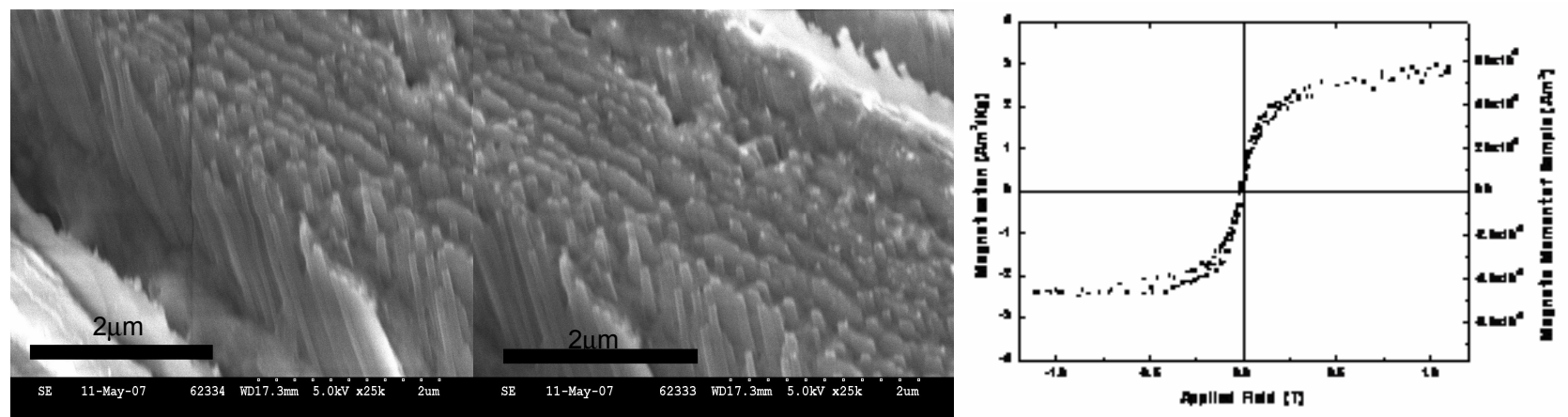

Figure 4: left; SEM images of standing arrays of polystyrene nanowires, right; VSM graph of maghemite loaded polystyrene
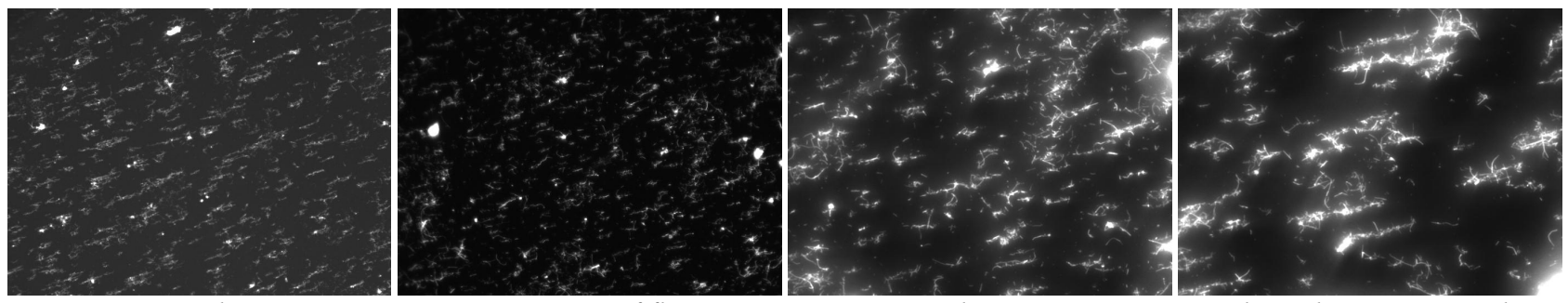

Figure 5: Fluorescent microscope images of fluorescent magnetic polystyrene nanowires aligned in an external magnetic field; from left magnification - x10, x20, x20 (increased lamp intensity), $x 40$

\section{Conclusions}

It has been shown here that the template synthesis for producing nanowires using a vacuum assisted infiltration of solutions is successful. The use of silica sol-gels has been demonstrated to produce undoped luminescent silica nanowires via carbon defect centres. Also magnetic silica nanowires have been produced by loading the sol-gel with iron oxide nanoparticles and shown to have potential future use in bio-medical applications via functionality of there surfaces and magnetic properties. Polystyrene has also been utilised to fabricate fluorescent magnetic nanowires via the template synthesis which show strong luminescence and useful magnetic properties.

\section{Acknowledgements}

This work was supported by Enterprise Ireland and Science Foundation Ireland (SFI).

\section{References}

1 Song, G., She, X., Fu, Z., and Li, J.: 'Preparation of good mechanical property polystyrene nanotubes with array structure in anodic aluminum oxide template using simple physical techniques', J. Mat. Res., 2004, 19, (11), pp. 3324-3328

2 Steinhart, M., Wehrspohn, R.B., Gösele, U., and Wendorff, J.H.: 'Nanotubes by Template Wetting: A Modular Assembly System', Angewandte Chemie International Edition, 2004, 43, (11), pp. 1334-1344 
3 Tang, Z., and Kotov, N.A.: 'One-Dimensional Assemblies of Nanoparticles: Preparation, Properties, and Promise', Adv. Mat., 2005, 17, (8), pp. 951-962

4 Bulte, J.W.M.: 'Magnetic nanoparticles as markers for cellular MR imaging', J Magn Magn Mater, 2005, 289, pp. $423-427$

5 Hultgren, A., Tanase, M., Chen, C.S., Meyer, G.J., and Reich, D.H.: 'Cell manipulation using magnetic nanowires', J Appl Phys, 2003, 93, pp. 7554 - 7556

6 Prina-Mello, A., Diao, Z., and Coey, J.: 'Internalization of ferromagnetic nanowires by different living cells', Journal of Nanobiotechnology, 2006, 4, (1), pp. 9

7 Nicewarner-Pena, S.R., Freeman, R.G., Reiss, B.D., He, L., Pena, D.J., Walton, I.D., Cromer, R., Keating, C.D., and Natan, M.J.: 'Submicrometer Metallic Barcodes', Science, 2001, 294, (5540), pp. 137-141

8 Hultgren, A., Tanase, M., Felton, E.J., Bhadriraju, K., Salem, A.K., Chen, C.S., and Reich, D.H.:

'Optimization of Yield in Magnetic Cell Separations Using Nickel Nanowires of Different Lengths', Biotechnol Prog, 2005, 21, pp. $509-515$

9 Pankhurst, Q.A., Connolly, J., Jones, S.K., and Dobson, J.: ‘Applications of magnetic nanoparticles in biomedicine', J Phys D Appl Phys, 2003, 36, pp. R167 - R181

10 Yang, H.H., Zhang, S.Q., Chen, X.L., Zhuang, Z.X., Xu, J.G., and Wang, X.R.: 'Magnetite-Containing Spherical Silica Nanoparticles for Biocatalysis and Bioseparations', Anal. Chem., 2004, 76, (5), pp. 1316-1321

11 Green, W.H., Le, K.P., Grey, J., Au, T.T., and Sailor, M.J.: 'White Phosphors from a Silicate-Carboxylate SolGel Precursor That Lack Metal Activator Ions', Science, 1997, 276, (5320), pp. 1826-1828

12 Y. P. Rakovich, Balakrishnan, S., Donegan, J.F., Perova, T.S., Moore, R.A., and Gun'ko, Y.K.: 'The

Fabrication, Fluorescence Dynamics, and Whispering Gallery Modes of Aluminosilicate Microtube Resonators', Adv. Func. Mat., 2007, 17, (7), pp. 1106-1114

13 Balakrishnan, S., Gun'ko, Y., Rakovich, Y.P., Donegan, J.F., Perova, T.S., and Moore, R.A.: 'Confined optical modes and amplified spontaneous emission from a microtube cavity formed by vacuum assited filtration', Appl. Phys. Lett., 2006, 89, pp. 143113

14 Son, S.J., Reichel, J., He, B., Schuchman, M., and Lee, S.B.: 'Magnetic Nanotubes for Magnetic-FieldAssisted Bioseparation, Biointeraction, and Drug Delivery’, J. Am. Chem. Soc., 2005, 127, (20), pp. 7316-7317 